\title{
On the Divisibility of an Odd Perfect Number by the Sixth Power of a Prime
}

\author{
By Wayne L. McDaniel
}

\begin{abstract}
It is shown that any odd perfect number less than $10^{9118}$ is divisible by the sixth power of some prime.
\end{abstract}

One of the oldest unsolved problems in mathematics is the problem of whether there exists an odd perfect number, i.e., an odd positive integer $n$ whose positive divisor sum $\sigma(n)$ is $2 n$.

It is well known that if such a number exists, it has the form

$$
n=p^{\alpha} \prod_{i=1}^{t} q_{i}^{2 \beta_{i}}
$$

where $t \geqq 5, p, q_{1}, q_{2}, \cdots, q_{t}$ are distinct primes, and $p \equiv \alpha \equiv 1(\bmod 4)($ see [3] for a summary of results on the existence of odd perfect numbers). It has been shown in recent years that if $n$ is an odd perfect number, then not all of the even exponents $2 \beta_{i}$, $1 \leqq i \leqq t$, are equal to 2 [6] or 4 [1], and if $\beta_{i}=1$ or 2 for all $i$, then $\beta_{i}=2$ for at least three values of $i[2]$.

We have examined the possibility that an odd perfect number exists for which $\beta_{i}<3$ for all $i, 1 \leqq i \leqq t$, and determined that no such number containing a prime less than 101 exists. This implies that any odd perfect number less than $10^{911}$ is divisible by the sixth power of some integer.

The investigation proceeds from the elementary observation that an odd prime power $P^{s}$ is a divisor of the odd perfect number $n$ iff $P^{s}$ is a divisor of $\sigma(n)$, takes note of the fact that $\sigma\left(P^{s}\right)=\prod_{m} F_{m}(P)$, where $F_{m}$ is the $m$ th cyclotomic polynomial and $m$ ranges over the divisors other than 1 of $s+1$, and utilizes the following well-known results of Kronecker (see [4]) to obtain additional prime factors of $n$ :

(1) If $q^{\gamma} \cdot d=m, q$ prime and not a divisor of $d$, then $q \mid F_{m}(P)$ iff $q \equiv 1(\bmod d)$ and $P$ belongs to $d(\bmod q)$;

(2) If $q \mid m$ and $q \mid F_{m}(P)$, then $q^{2} \nmid F_{m}(P)$, provided $m>2$;

(3) If $k \nmid m$ and $k \mid F_{m}(P)$, then $k \equiv 1(\bmod m)$.

All factoring was accomplished and checked on an electronic programable calculator and an IBM 1130 computer.

In this paper, we assume $n=p^{\alpha} q_{1}^{2 \beta_{1}} q_{2}^{2 \beta_{2}} \cdots q_{t}^{2 \beta_{t}}, 2 \beta_{i}<6, p \equiv \alpha \equiv 1(\bmod 4)$, and use the notation $P^{s} \| n$ to mean that $P^{s} \mid n$, but $P^{s+1} \nmid n$. Because the proofs are largely computational we do not include the details of all cases in this paper (a complete proof will be supplied by the author upon request). For the benefit of the novice in odd perfect number theory, we present the proof, which is typical, of the following

LEMMA. If $n$ is perfect, $n$ is not divisible by $3 \cdot 11$.

Received December 3, 1969, revised September 14, 1970.

AMS 1970 subject classifications. Primary 10A40; Secondary 10A20, 10 A05.

Key words and phrases. Perfect number, cyclotomic polynomial.

Copyright (c) 1971, American Mathematical Society 
Proof. We suppose $3^{b} \| n$ and $11^{c} \| n$, and distinguish two cases.

Case 1. $b$ and $c$ not both equal to 4 . If $b=c=2$, then $\sigma\left(3^{2} \cdot 11^{2}\right)=13 \cdot 7 \cdot 19 \mid n$, so $n$ is divisible by $3^{2} \cdot 11^{2} \cdot 13 \cdot 7^{2} \cdot 19^{2}$, from which it follows that

$$
\sigma(n) / n \geqq \sigma\left(3^{2} \cdot 11^{2} \cdot 13^{1} \cdot 7^{2} \cdot 19^{2}\right) /\left(3^{2} \cdot 11^{2} \cdot 13^{1} \cdot 7^{2} \cdot 19^{2}\right)>2
$$

and $n$ is not perfect. If $b=2$ and $c=4, \sigma\left(3^{2} \cdot 11^{4}\right)=13 \cdot 5 \cdot 3221 \mid n$ and, as above, $\sigma(n) / n>2$. If $b=4$ and $c=2, \sigma\left(3^{4} \cdot 11^{2}\right)=11^{2} \cdot 7 \cdot 19 \mid n$ and, again, $\sigma(n) / n>2$.

Case 2. $b=c=4$. $\sigma\left(3^{4} \cdot 11^{4}\right)=11^{2} \cdot 5 \cdot 3221 \mid n$. If $5^{2 \beta} \mid n$, for $\beta \geqq 1$, then $\sigma(n) / n \geqq$ $\sigma\left(3^{4} \cdot 5^{2} \cdot 11^{4}\right) /\left(3^{4} \cdot 5^{2} \cdot 11^{4}\right)>2$. We may assume, then, that $\beta=0$, i.e., $p=5$. Now, $3221^{4} \nmid n$, since $5 \mid \sigma\left(3221^{4}\right)$ and $5 \mid \sigma\left(11^{4}\right)$; so $\sigma\left(3221^{2}\right)=10378063$ divides $n$.

2a. $10378063^{2} \| n . \sigma\left(10378063^{2}\right)$ is divisible by 769 . Now, $769^{2} \nmid n$, since $31 \mid \sigma\left(769^{2}\right)$ and $\sigma\left(3^{4} \cdot 11^{4} \cdot 5 \cdot 31\right)>2\left(3^{4} \cdot 11^{4} \cdot 5 \cdot 31\right)$. So, $769^{4} \| n$, which implies that 541 , a factor of $\sigma\left(769^{4}\right)$, divides $n$. But if $541^{2} \| n$, then 7 , a factor of $\sigma\left(541^{2}\right)$, divides $n$; this is impossible since $\sigma\left(3^{4} \cdot 5 \cdot 7^{2}\right) /\left(3^{4} \cdot 5 \cdot 7^{2}\right)>2$. On the other hand, if $541^{4} \| n$, then $5^{2} \mid n$, contrary to our assumption. Hence, $10378063^{2} \nmid n$.

2b. $10378063^{4} \| n \cdot \sigma\left(10378063^{4}\right)$ is divisible by 33151 . Since $5^{2} \nmid n, 33151^{4} \not n$, so $\sigma\left(33151^{2}\right)=3 \cdot 366340651 \mid n$. This latter factor is prime, and must occur to the 2 nd power as a factor of $n$. The prime 68409301 divides $\sigma\left(366340651^{2}\right)$ and $7 \mid \sigma\left(68409301^{2}\right)$. However, this implies $3^{4} \cdot 5 \cdot 7^{2} \mid n$, which implies $\sigma(n) / n>2.68409301^{4} \not \mid n$ since $5^{2} \nmid n$.

It is now immediate that $3^{4} \nVdash n$, since $11 \mid \sigma\left(3^{4}\right)$. That $3^{2} \nVdash n$ is shown in

THEOREM 1. $n$ is not perfect if $3 \mid n$.

If, now, $n$ is an odd perfect number, Theorem 1 implies that $\left(F_{2}(p)\right) / 2=$ $(p+1) / 2 \not \equiv 0(\bmod 3)$, so $p \equiv 1(\bmod 3) ;$ since, also, $p \equiv 1(\bmod 4)$, we have $p \equiv$ 1 (mod 12). Relying heavily on this fact, Theorem 1 , and the fact that $11 \mid \sigma\left(q^{4}\right)$ iff $q \equiv$ $3,4,5,9(\bmod 11)$, we are able to prove

THEOREM 2. $n$ is not perfect if $n$ is divisible by 5 .

Having found that neither 3 nor 5 is a factor of $n$, we now find it an easy matter to show that the smallest prime factor of $n$ must be fairly large. We establish

THEOREM 3. $n$ is not perfect if $n$ has a prime factor less than 101.

Proof. Let $q$ denote the least prime factor of $n$, and suppose $q<101$. We note that $p \geqq 2 q-1$, since $(p+1) / 2$ divides $n$ and is therefore greater than or equal to $q$; hence $q^{2} \| n$ or $q^{4} \| n$.

Suppose $q^{2} \| n$. Then $\sigma\left(q^{2}\right)$ is prime, and it follows that $q=17,41,59,71$, or 89 . If $q=17$, then $n=17^{2} \cdot 307^{4} \cdot 1051^{\circ} \ldots$, which is impossible for $e=2$ or 4 . If $q=41$, then $n=41^{2} \cdot 1723^{4} \cdot 6101^{2} \cdot 7 \cdots$, which is not possible since $7<q$. $q$ may not equal 59 , since $3541 \mid \sigma\left(59^{2}\right)$ and $7 \mid \sigma\left(3541^{\alpha}\right)$. If $q=71, n=71^{2} \cdot 5113^{\alpha} \cdot 2557^{4} \cdot 11 \cdots$, or $n=71^{2} \cdot 5113^{4} \cdot 11 \cdots$, and if $q=89$, then $n$ is divisible by $8011^{2}$ or $8011^{4}$, neither of which is possible.

Suppose $q^{4} \| n$. Since $5 \mid \sigma\left(q^{4}\right)$ for $q \equiv 1(\bmod 5)$, and $11 \mid \sigma\left(q^{4}\right)$ for $q \equiv 3,4,5,9$ $(\bmod 11), q$ must be one of the primes $7,13,17,19,23,29,43,67,73,79,83,89$. If $Q$ is a prime factor of $\sigma\left(q^{4}\right)$, then $Q \equiv 1(\bmod 5)$; it follows that $\sigma\left(Q^{2}\right) \mid n$ or, if $Q \equiv$ $1(\bmod 12),[(Q+1) / 2] \mid n$. For each of the above values of $q$, other than $7,13,17$, 29, 67 and 83, there exists a prime factor $Q$ of $\sigma\left(q^{4}\right)$ such that $\sigma\left(Q^{2}\right)$ and (if $Q \equiv$ $1(\bmod 12))(Q+1) / 2$ have a divisor less than $q$. For these remaining six values of $q$, we proceed as in Lemma 1 , readily obtaining in each case the same conclusion, that is, that $n$ has a divisor less than $q$. 
We remark, in passing, that the impossibility of factors of $n$ less than 101 , and in particular 3 and 5 , imposes several conditions on $p^{\alpha}$. One may easily show, for example, that $p \geqq 673, p \equiv 1,13$ or $37(\bmod 60)$, and $\alpha \equiv 1$ or $9(\bmod 12)$. This last condition implies the result of Kanold [2] that $n=p^{5} \prod_{i=1}^{t} q_{i}^{2 \beta_{i}}$, for $2 \beta_{i}=2$ or 4 , is not a perfect number.

THEOREM 4. If an odd perfect number $N$ exists, then either $N$ is divisible by (at least) the sixth power of a prime, or $N>10^{9118}$.

Proof. Theorem 3 proves that if $N$ is not divisible by the sixth power of a prime then the least prime factor of $N$ is $\geqq 101$. Karl Norton's paper [5] contains a table which shows that any odd perfect number whose least prime factor is $\geqq 101$ has at least 1331 distinct prime factors and has as its largest prime factor a number $\geqq 11197$. Letting $P_{r}=101$ and $P_{s}=11197$, the inequality (see [5, p. 369])

$\log N>2 P_{s}\left(1-\frac{1}{2 \log P_{s}}\right)-2 P_{r}\left(1+\frac{1}{2 \log P_{r}}\right)$ $+6 \log P_{r}+2 \log P_{r+1}-\log P_{s}$

yields the lower bound $N>10^{9118}$.

Department of Mathematics

University of Missouri-St. Louis

St. Louis, Missouri 63121

1. H.-J. KANOLD, "Untersuchen über ungerade vollkommene Zahlen," J. Reine Angew. Math., v. 183, 1941, pp. 98-109. MR 3, 268.

2. H.-J. KANOLD, "Einige neuere Bedingungen für die Existenz ungerader vollkommener Zahlen," J. Reine Angew. Math., v. 192, 1953, pp. 24-34. MR 15, 854.

3. P. J. MCCARTHY, "Odd perfect numbers," Scripta Math., v. 23, 1957, pp. 43-47. MR 21 \#21.

4. T. NAGELl, Introduction to Number Theory, Wiley, New York, 1951, pp. 156-168. MR 13, 207.

5. K. K. NorTon, "Remarks on the number of factors of an odd perfect number," Acta. Arith., v. 6, 1960/61, pp. 365-374. MR 26 \#4950.

6. R. STEUERWALD, "Verschärfung einer notwendigen Bedingung für die Existenz einer ungeraden vollkommenen Zahl," S.-B. Math.-Nat. Abt. Bayer. Akad. Wiss., 1937, pp. 68-73. 